PPPL-3456

PPPL-3456

UC-70

Numerical Study of Tilt Stability of Prolate Field-reversed Configurations

by

E. V. Belova, S. C. Jardin, H. Ji, M. Yamada, and R. Kulsrud

June 2000

$\int \frac{D}{5}[\sqrt{\zeta}] \int \Omega \begin{aligned} & \text { PAINCETON } \\ & \text { PLABMA PHYSICS } \\ & \text { LABOAATOAY }\end{aligned}$

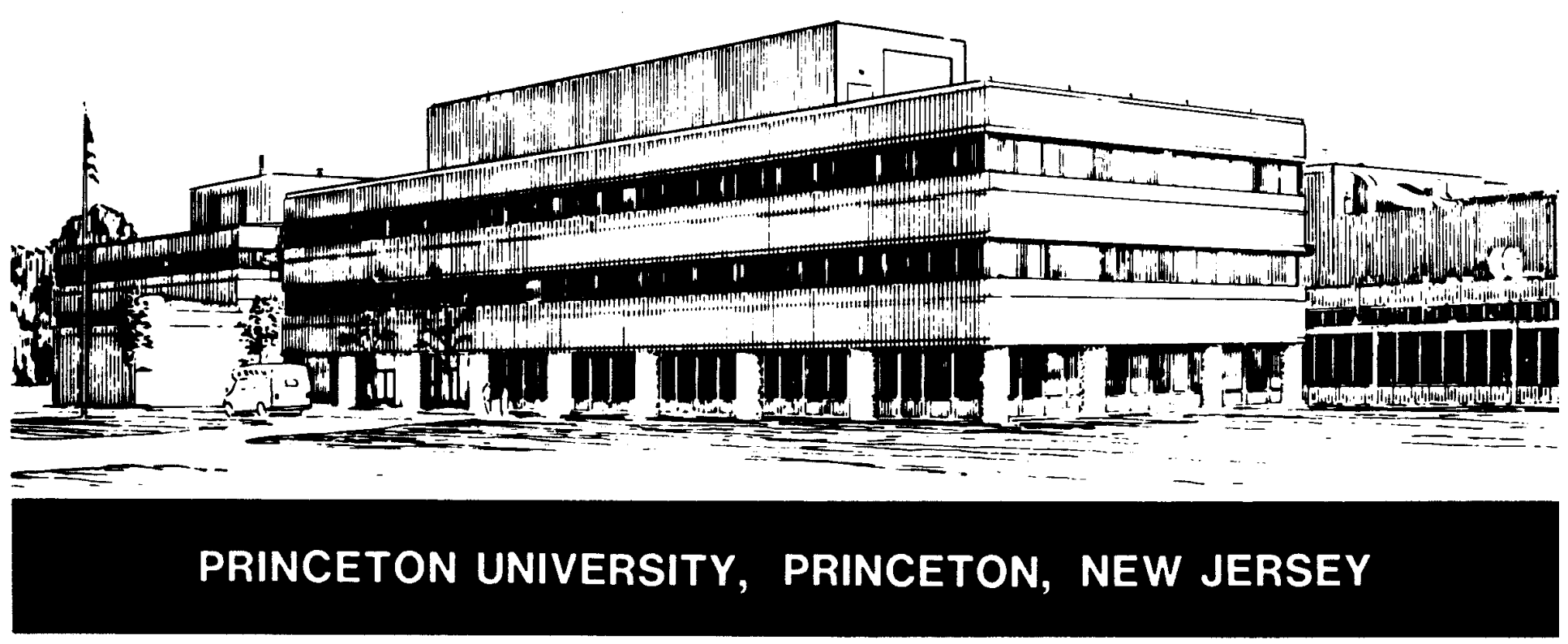




\section{PPPL Reports Disclaimer}

This report was prepared as an account of work sponsored by an agency of the United States Government. Neither the United States Government nor any agency thereof, nor any of their employees, makes any warranty, express or implied, or assumes any legal liability or responsibility for the accuracy, completeness, or usefulness of any information, apparatus, product, or process disclosed, or represents that its use would not infringe privately owned rights. Reference herein to any specific commercial product, process, or service by trade name, trademark, manufacturer, or otherwise, does not necessarily constitute or imply its endorsement, recommendation, or favoring by the United States Government or any agency thereof. The views and opinions of authors expressed herein do not necessarily state or reflect those of the United States Government or any agency thereof.

\section{Availability}

This report is posted on the U.S. Department of Energy's Princeton Plasma Physics Laboratory Publications and Reports web site in Calendar Year 2000. The home page for PPPL Reports and Publications is: http://www.pppl.gov/pub_report/

DOE and DOE Contractors can obtain copies of this report from:

U.S. Department of Energy

Office of Scientific and Technical Information

DOE Technical Information Services (DTIS)

P.O. Box 62

Oak Ridge, TN 37831

Telephone: (865) 576-8401

Fax: (865) 576-5728

Email: reports@adonis.osti.gov

This report is available to the general public from:

National Technical Information Service

U.S. Department of Commerce

5285 Port Royal Road

Springfield, VA 22161

Telephone: $1-800-553-6847$ or

(703) $605-6000$

Fax: (703) 321-8547

Internet: http://www.ntis.gov/ordering.htm 


\title{
NUMERICAL STUDY OF TILT STABILITY OF PROLATE FIELD-REVERSED CONFIGURATIONS
}

\author{
E. V. Belova, S. C. Jardin, H. Ji, M. Yamada, R. Kulsrud \\ Princeton Plasma Physics Laboratory, Princeton, NJ 08543, USA
}

\begin{abstract}
Global stability of the Field-Reversed Configuration (FRC) has been investigated numerically using both 3D MHD and hybrid (fluid electron and $\delta f$ particle ion) simulations. The stabilizing effects of velocity shear and large ion orbits on the $n=1$ internal tilt mode in the prolate FRCs have been studied. Sheared rotation is found to reduce the growth rate, however a large rotation rate with Mach number of $M \gtrsim 1$ is required in order for significant reduction in the instability growth rate to occur. Kinetic effects associated with large thermal ion orbits have been studied for different kinetic equilibria. Our simulations show that there is a reduction in the tilt mode growth rate due to finite ion Larmor radius (FLR) effects, but complete linear stability has not been found, even when the thermal ion gyroradius is comparable to the distance between the field null and the separatrix. The instability existing beyond the FLR theory threshold could be due to the resonant interaction of the wave with ions whose Doppler shifted frequency matches the betatron frequency.

PACS numbers: 52.35.Py, 52.55.Hc, 52.65.Rr, 52.65.Kj
\end{abstract}




\section{INTRODUCTION}

The Field-Reversed Configuration (FRC) is a compact toroid with negligible toroidal field, in which plasma is confined by a poloidal magnetic field associated with toroidal diamagnetic current carried by the plasma. The FRC offers a unique fusion reactor potential because of its compact and simple geometry, translation properties, and high plasma beta. Although many MHD modes are predicted to be unstable, prolate FRCs have been produced successfully by several formation techniques and show surprising macroscopic resilience ${ }^{1}$.

A substantial literature exists of FRC stability studies in both the MHD (ie, fluid) and kinetic (ie, particle ion) descriptions ${ }^{2-9}$. The $n=1$ tilt instability is thought to be the most dangerous MHD instability because it is both strongly growing and a global mode that is not readily stabilized when kinetic effects are included. This mode is internal to the magnetic separatrix in the prolate FRC and thus cannot be effectively stabilized by external means. MHD simulations show that the tilt mode growth rate is on the order of the inverse Alfvén transit time and depends only weakly on the equilibrium profiles (within a factor of two). Plasma rotation and the inclusion of the Hall term have been considered as stabilizing mechanisms for this mode and were found to reduce the growth rate $^{2}$. However a change in the linear mode structure prevented the complete stabilization that was predicted earlier based on a trial function dispersion analysis. In MHD calculations including the effect of gyro-viscosity ${ }^{5}$, up to a factor of 3 reduction in growth rate has been found, but not complete stability. It has also been found that there is no nonlinear saturation of the $n=1$ tilt instability in the MHD description ${ }^{2,7}$.

The key parameters related to the finite Larmor radius (FLR) stability of the FRC are $\bar{s}$, which measures the number of thermal ion gyro-radii in the configuration and $E$, the separatrix elongation. These are defined by

$$
\bar{s}=\int_{R_{0}}^{R_{s}} \frac{r d r}{R_{s} \rho_{i}},
$$

and $E=Z_{s} / R_{s}$. Here $R_{0}$ and $R_{s}$ are the magnetic null and the separatrix radius at the midplane, $\rho_{i}$ is the local ion Larmor radius, and $Z_{s}$ is the separatrix half-length at $r=0$. The kinetic parameter $\bar{s}$ indicates the importance of finite Larmor radius (FLR) effects, which are strong for $\bar{s} \sim 1$, while the $\bar{s} \gg 1$ (small Larmor radius) limit corresponds to the 
MHD regime. It has been found in a number of studies that the tilt mode growth rate is reduced with increasing elongation (for a fixed separatrix radius). It is inversely proportional to $E$ for an equilibrium with an elliptic separatrix shape, but this dependence is weaker for a racetrack separatrix shape ${ }^{3}$. Another useful stability parameter is the ratio $\bar{s} / E$, which arises from FLR theory ${ }^{4,10}$, according to which FLR effects are important when $\omega^{*} \gtrsim \gamma$. Here $\omega^{*}$ is the diamagnetic frequency, $\gamma=C V_{A} / Z_{s}$ is the tilt mode growth rate in the MHD approximation, $V_{A}$ is a characteristic Alfvén velocity, and $C$ is a coefficient of the order of unity. From these relations, an approximate condition for FLR effects to be important is: $\bar{s} / E \lesssim 0.2-0.5$.

Early linear kinetic calculations using a Vlasov-fluid trial function approach ${ }^{6}$ have found a greatly reduced growth rate for $\bar{s} \approx 2-3$ and even predicted complete stabilization at $\bar{s} \lesssim 1.5(E=7.7)$. However, in a later spectral stability study with a more general set of basis functions ${ }^{9}$, complete stabilization of the tilt mode has not been found, even for very small values of $\bar{s}$.

Kinetic calculations using the initial value particle simulation approach have been performed for small elongation $(E \leq 2.5)$ and moderate kinetic regimes with $1.6 \leq \bar{s} \leq 12$. These were therefore in the parameter range $(\bar{s} / E \gtrsim 1)$ for which no significant stabilization is predicted by the FLR theory. Simulation studies for equilibria with $E=2-2.5$ and $\bar{s} \gtrsim 2$ have indeed found the tilt mode growth rate to be close to that predicted by MHD, and only slightly affected by the $\bar{s}$ value in this range ${ }^{11,12}$.

Several other effects have been identified in these simulation studies as possible stabilizing effects. It was found in one case that stability depends on the value of the plasma beta at the magnetic separatrix ${ }^{11}, \beta_{s}$. In another paper $^{12}$, a spontaneously generated toroidal magnetic field has been proposed as being a stabilizing factor. An earlier particle simulation study ${ }^{13}$ considered the tilt stability for $E=1.5$ and two values of $\bar{s}, 1.6$ and 12 , and found an order of magnitude growth rate reduction in the first case. However, since their simulation run time was several times shorter than an estimated growth time, this result may require further verification. The effect of energetic ion beams on the tilting instability was studied by Barnes and Milroy ${ }^{14}$ using a hybrid MHD/particle simulation, and was found to provide significant stabilization of the tilt mode for a beam energy of about $40 \%$ of the total energy 
and beam density larger than $1.5 \%$ of the bulk density.

Experimentally, it seems clear that global stability to the $n=1$ tilt has been achieved, at least in some parameter regimes. Most FRC experiments have been performed with relatively large elongation, $E \sim 5-9$, and small to moderate $\bar{s}(\bar{s} \sim 1-8)$. In most cases, FRCs with small $\bar{s}$ values $(\bar{s} \lesssim 2)$ appear to be stable, and have been observed to persist for over 100 Alfvén times ${ }^{15,16}$. Some $n=1$ activity is often observed during or shortly after the formation phase of a FRC, but the origin of this has not been positively identified. This apparent stability is inconsistent with the MHD predictions and may indicate that kinetic effects are important and account for the observed FRC stability. Some experimental studies have reported a correlation between the appearance of tilt instabilities and a degradation of confinement at larger values of $\bar{s}$ and smaller elongation ${ }^{15}(\bar{s} / E \sim 1)$. This further supports the FLR stabilization mechanism theory. The data from the Large-s Experiment (LSX), on the other hand, seems to indicate no such correlation, and good confinement over a large range of $\bar{s}(1 \lesssim \bar{s} \lesssim 8)$ has been reported ${ }^{16,17}$. However, common to all the experimental studies is the difficulty in FRC formation at large $\bar{s}$. Although in some cases this was attributed to formation inadequacies ${ }^{16}$, the possibility that it is related to a reduction in stabilizing factors for the tilt instability at large $\bar{s}$ can not be ruled out. We note finally that there are experimental difficulties in detecting the tilt instability in the FRC, related to its internal nature and its lack of a clear signature on the axial interferometry diagnostic. This situation is in contrast to that of spheromak experiments, where the tilting modes have been clearly observed in several devices ${ }^{18,19}$. These experiments have shown that the spheromak tilt mode growth rate is reduced significantly when the plasma shape is oblate $(E<1)$, and that it can be stabilized completely by close-fitting figure 8 coils $^{20}$.

In the present paper, we attempt to unify and extend the previous simulation studies by presenting the results of extensive and systematic studies of prolate FRC stability in which we employ both 3D MHD and hybrid (fluid electron and particle ion) simulations. In particular, the stabilizing effects of velocity shear and finite ion Larmor radius on the $n=1$ tilt mode have been examined (this paper addresses only the prolate FRC for which the elongation $E>1$, the stability of the oblate $\mathrm{FRC}$ for which $E \leq 1$ will be addressed in a future publication). The new 3D nonlinear MHD/particle code developed for this stability 
study is described in the next section. In contrast to previous work, the $\delta f$ method $^{21}$ has been used to reduce numerical noise in the particle simulations. Equations of the $\delta f$ scheme are presented and the solution of the equilibrium problem with kinetic ions and sheared rotation is discussed. Results of the MHD simulations including sheared flows, are described in Sec. III. In Sec. IV the hybrid simulations results are presented for different kinetic equilibria. Conclusions and a discussion are given in Sec. V.

\section{NUMERICAL SCHEME AND SIMULATION MODELS}

In our FRC global stability code ${ }^{22}$ two different physical models have been implemented: a 3D nonlinear one-fluid MHD model, and a 3D nonlinear hybrid scheme with particle ions and fluid electrons.

\section{A. MHD model}

In the MHD version of the code, the nonlinear resistive one-fluid MHD equations are advanced using a second order explicit time stepping scheme with forth-order accurate spatial derivatives:

$$
\begin{gathered}
\frac{\partial \rho}{\partial t}+\nabla \cdot(\rho \mathbf{v})=0 \\
\frac{\partial \rho \mathbf{v}}{\partial t}=-\nabla \cdot(\rho \mathbf{v} \mathbf{v})-\nabla p+\mathbf{J} \times \mathbf{B} / c+\mu \Delta \mathbf{v}, \\
\partial p^{1 / \gamma} / \partial t+\nabla \cdot\left(\mathbf{v} p^{1 / \gamma}\right)=\frac{(\gamma-1)}{\gamma} p^{1 / \gamma-1}\left[\eta J^{2}+\mu(\nabla \times \mathbf{v})^{2}+\mu(\nabla \cdot \mathbf{v})^{2}\right], \\
\partial \mathbf{A} / \partial t=-c \mathbf{E}, \\
\mathbf{E}=-\mathbf{v} \times \mathbf{B} / c+\eta \mathbf{J} .
\end{gathered}
$$

Here $\rho$ and $p$ are the plasma density and pressure, $\mathbf{v}$ is the fluid velocity, $\mathbf{A}$ is the vector potential, $\mathbf{B}=\nabla \times \mathbf{A}$ is the magnetic field, $\mathbf{J}=c /(4 \pi) \nabla \times \mathbf{B}$ is the total current, $\mu$ is an artificial viscosity coefficient, and an adiabatic equation of state is used.

In the FRC stability studies, a cylindrical grid is employed and the finite difference approximation is used in all three directions $(r, \phi, z)$. A fine resolution of $(100 \times 32 \times 150)$ grid 
points has typically been used in our MHD runs. Periodic boundary conditions are applied in both the toroidal and axial directions, while a perfectly conducting boundary is assumed at the cylindrical wall $r=R_{c}$ outside the separatrix and geometric boundary conditions are implemented at $r=0$. The cylinder length is taken to be large enough, so that periodic boundary conditions in the axial direction have no effect on the stability. The conservative form of the equations and the time-centered trapezoidal leap-frog scheme ${ }^{23}$ used for the time evolution ensure very good energy conservation, with the change in the total energy being less than $0.1 \%$ of the perturbation energy in the production runs. Hyperresistivity in the induction equation and artificial viscosity in the momentum equation are added for numerical stability in the nonlinear runs.

The vacuum region outside the separatrix is treated in the same way as the plasma region, except that a small cutoff density (typically 0.05 to 0.1 fraction of the peak density) is applied. The density is not allowed to become smaller than the cutoff value in order to avoid the severe time step restrictions that the Courant condition would impose. In some cases a nonuniform resistivity profile with resistivity inversely proportional to the plasma density has been used, but the tilt mode stability was found to be insensitive to the resistivity profile.

The linearized form of the MHD equations (1)-(5) has also been implemented as an additional option, and this has been particularly useful for the linear stability studies of $n>1$ modes. The MHD version of the code has been benchmarked both by simulating normal modes in a cylinder, and by reproducing the previous initial value MHD simulations results $^{2,24}$. Very good agreement with the spectral stability study results ${ }^{3}$ has been obtained as well.

A 2D self-consistent equilibrium is found by solving a Grad-Shafranov equation which includes equilibrium flows, and this is used as an initial condition for the 3D stability calculations. An MHD equilibrium with sheared flow is obtained assuming constant temperature, $T=p / \rho$, in this case the pressure profile must be written as:

$$
p(\psi, r)=p_{0} g(\psi) \exp \left(\frac{m_{i} r^{2} \omega^{2}(\psi)}{2 T}\right),
$$

where $\omega(\psi)$ is the angular velocity. The Grad-Shafranov equation for the poloidal magnetic flux function becomes ${ }^{25}$ 


$$
r \frac{\partial}{\partial r}\left(\frac{1}{r} \frac{\partial \psi}{\partial r}\right)+\frac{\partial^{2} \psi}{\partial z^{2}}=-r^{2}\left[p_{0} \frac{\partial g}{\partial \psi}+p_{0} g \frac{m_{i} r^{2}}{T} \omega \frac{\partial \omega}{\partial \psi}\right] \exp \left(\frac{m_{i} r^{2} \omega^{2}}{2 T}\right) .
$$

Here we have defined $\psi$ by $\mathbf{B}=\nabla \phi \times \nabla \psi$ in the equilibrium. Eq. (7) is solved iteratively using the alternating direction implicit method (ADI) and applying a global constraint (total current, $I_{\phi}$ ) at each iteration as discussed, for example, by Hewett and Spencer ${ }^{26}$. An equilibrium solution is calculated in a cylinder with boundary conditions: $\psi=0$ at $r=0$, $\psi=\psi_{w}$ at $r=R_{c}$, and $\partial \psi / \partial z=0$ at $z= \pm Z_{c}$, where $R_{c}$ and $Z_{c}$ are the cylinder radius and half length respectively.

\section{B. Hybrid model}

A hybrid scheme with cold fluid electrons $\left(p_{e}=0\right)$ and particle ions is used in the kinetic simulations. The particle ion motion is described by the Lorenz force equations:

$$
\begin{gathered}
\frac{d \mathbf{x}}{d t}=\mathbf{v}, \\
\frac{d \mathbf{v}}{d t}=\frac{e}{m_{i}}(\mathbf{E}-\eta \mathbf{J}+\mathbf{v} \times \mathbf{B} / c),
\end{gathered}
$$

with the standard leapfrog scheme used for the time advance. The term $-\eta \mathbf{J}$ in Eq. (9) represents the collisional drag on the ions, and it is needed for momentum conservation. The electric field in the hybrid scheme is calculated from the electron momentum equation neglecting the inertial term:

$$
\mathbf{E}=-\mathbf{u}_{\mathbf{e}} \times \mathbf{B} / c+\eta \mathbf{J}
$$

where $\mathbf{u}_{\mathbf{e}}=-\left(\mathbf{J}-\mathbf{J}_{\mathbf{i}}\right) /\left(e n_{e}\right)$ is the electron fluid velocity, $\mathbf{J}_{i}$ is the ion current, and quasineutrality is assumed $\left(n_{e}=n_{i}\right)$. The magnetic field is calculated as in the MHD scheme, Eq. (4).

The fluid equations (4) and (10) are advanced on a finite-difference mesh in a cylindrical coordinate system, while the particle advance and current and density accumulation are done on a 3D Cartesian grid. Quadratic spline interpolation is used for mapping between the cylindrical and the Cartesian meshes, while linear spline interpolation is used in the particle loops (gather/scatter operations). Since the computational time required to advance the particles greatly exceeds the time needed to advance the field equations, subcycling is used to update the fields with small enough time step to avoid numerical instability at small $\bar{s}$. 
The particle coordinates and weight factors are advanced with a larger time step, which is limited by the ion cyclotron motion and is typically set to $d t=0.1 / \omega_{c i}$.

The $\delta f$ method $^{21}$ is utilized to reduce numerical noise in the simulations. In the $\delta f$ scheme the equilibrium distribution function is assumed to be known analytically, so the zeroorder density $n_{i 0}$ and current $\mathbf{J}_{i 0}$ can be calculated. The perturbed part of the distribution function is calculated along a set of characteristics (8), (9) by assigning a weight $w \sim \delta F$ to each simulation particle. The particle weights are then used to calculate the perturbed ion density $\delta n_{i}$ and current $\delta \mathbf{J}_{i}$. Since the particle weight is no longer a constant of the motion (as in the conventional particle simulation method), the evolution equation for the weight has to be added to the equations of motion (8) and (9):

$$
\frac{d w}{d t}=-\left(\frac{F}{P}-w\right) \frac{d F_{0}}{d t} \frac{1}{F_{0}}
$$

where $w=\delta F / P, F$ is the ion distribution function, and $P$ is the distribution function of the simulation (marker) particles. In the simulations $\delta F$ can be approximated by the weighted Klimontovich distribution

$$
\delta F=\sum_{m} w_{m} \delta\left(\mathbf{x}_{m}-\mathbf{x}\right) \delta\left(\mathbf{v}_{m}-\mathbf{v}\right)
$$

and the perturbed ion density and current density are calculated as follows

$$
\begin{gathered}
\delta n_{i}(\mathbf{x})=\sum_{m} w_{m} S\left(\mathbf{x}_{m}-\mathbf{x}\right), \\
\delta \mathbf{J}_{i}(\mathbf{x})=\sum_{m} w_{m} \mathbf{v}_{m} S\left(\mathbf{x}_{m}-\mathbf{x}\right),
\end{gathered}
$$

where $S$ is the (linear) shape function. In all the calculations described in this work, the simulation particles were initially loaded in phase space according to the physical distribution function, $F_{0}$, which corresponds to setting $F / P=1$ in Eq. (11).

The equilibrium ion distribution function $F_{0}$ is a function of the constants of motion, which in the case of an azimuthally symmetric equilibrium, are the particle energy, $\varepsilon=$ $m_{i} v^{2} / 2+e \varphi$, and the azimuthal angular momentum, $p_{\phi}=m_{i} r v_{\phi}-e \psi$, where $\psi=-r A_{\phi}$ is the poloidal magnetic flux function, and $\varphi$ is the equilibrium electrostatic potential. For $F_{0}=$ $F_{0}\left(\varepsilon, p_{\phi}\right)$ the time advancing of the particle weights requires calculation of time derivatives of $\varepsilon$ and $p_{\phi}$ : 


$$
\begin{aligned}
\frac{d \varepsilon}{d t} & =e \mathbf{v} \cdot(\delta \mathbf{E}-\eta \delta \mathbf{J}) \\
\frac{d p_{\phi}}{d t} & =\operatorname{er}\left[\left(\delta E_{\phi}-\eta \delta J_{\phi}\right)+\mathbf{v} \times \delta \mathbf{B} \cdot \hat{\phi}\right] .
\end{aligned}
$$

Two classes of kinetic equilibria were studied in the simulations presented here. The first class has zero electron current and no electric field. All current is carried by the ions and an exponential rigid-rotor distribution function is used:

$$
\begin{aligned}
F_{0} & =F_{0}\left(\varepsilon-\Omega p_{\phi}\right) \\
& =n_{0}\left(m_{i} / 2 \pi T_{i}\right)^{3 / 2} \exp \left(-\varepsilon / T_{i}+\Omega p_{\phi} / T_{i}\right)
\end{aligned}
$$

where $\Omega$ is the constant angular rotation frequency, and $T_{i}$ is the uniform ion temperature. It can be shown that this distribution function corresponds to a local shifted Maxwellian distribution with the ion density given by

$$
n_{i}(\mathbf{x})=n_{0} \exp \left(\frac{m_{i} r^{2} \Omega^{2}}{2 T_{i}}-\frac{e \psi \Omega}{T_{i}}\right)
$$

and mean ion velocity $V_{\phi}=r \Omega$. The force balance for this equilibrium can be written as:

$$
-m_{i} n_{i} r \Omega^{2} \hat{\mathbf{r}}=-T_{i} \nabla n_{i}+\mathbf{J}_{i} \times \mathbf{B} .
$$

For the exponential rigid-rotor distribution function (18), we were not able to find an equilibrium with the separatrix elongation larger than $E=2$, probably due to the exponential dependence of plasma pressure on $\psi$. In the simulations with larger elongations, the second class of the kinetic equilibria has been used. The second class of equilibrium distribution functions is $F_{0}=F_{0}(\varepsilon)$, with $\mathbf{J}_{i}=0$ and all current is carried by the electrons. From the ion momentum equation we have

$$
0=-T_{i} \nabla n_{i}+e n_{i} \mathbf{E}
$$

where $\mathbf{E}=\mathbf{J} \times \mathbf{B} /\left(e n_{e}\right)$ is an equilibrium electric field.

Since in both cases the ion pressure is a scalar, Eq. (7) has been used to find an equilibrium solution.

\section{MHD SIMULATIONS}




\section{A. Profile effects}

Numerous MHD calculations (both linearized and nonlinear) have shown the $n=1$ tilt mode to be strongly unstable with growth rate on the order of the inverse Alfvén time (without rotation)

$$
\gamma=C V_{A} / Z_{s}=C V_{A} /\left(R_{s} E\right),
$$

and found no nonlinear stabilization ${ }^{2,7}$. Here $V_{A}$ is a characteristic Alfvén velocity, defined by $V_{A}=B_{0} / \sqrt{4 \pi \rho_{0}}$, where $B_{0}$ is an external magnetic field at the midplane, $\rho_{0}$ is the plasma density at the magnetic null, and $C$ is the coefficient of the order of unity. The most detailed linear MHD stability analysis employing the spectral code by Iwasawa et al. ${ }^{3}$ considered the effects of elongation, current profile and the magnetic separatrix shape on the $n=1$ and $n>1$ modes with both even and odd symmetry (symmetry is defined in terms of $v_{r}$ symmetry relative to the midplane, $z=0$ ). It was shown that the previously believed inverse scaling of the tilt mode growth rate with elongation, Eq. (20), applies only to the special case of the equilibrium with an elliptical separatrix and a flat current profile. For a racetrack separatrix and large enough elongation $(E \gtrsim 3)$, the tilt (odd) mode growth rate has been found to be largely independent of $E$, and another $n=1$ mode, with even symmetry relative to the midplane, has been shown to be unstable with a growth rate close to that of the tilt mode. This result can be easily understood, if one considers the linear mode structures for the elliptical and racetrack equilibria.

Figure 1 shows velocity vector plots produced at the linear phase of the MHD simulations with different separatrix shapes and initial perturbations of the odd and even symmetry. In agreement with the results of Iwasawa et al. ${ }^{3}$, our simulations show that for a racetrack equilibrium (Fig. 1b,c) the perturbation is localized near the end regions, where the curvature is large, and the structure of the $n=1$ even mode is very similar to that of the odd (tilt) mode, except for the direction of the velocity vectors (Fig. 1c). The growth rates of these two modes are nearly identical as well, and do not change significantly as the elongation increases. This behavior implies that the perturbation growths at the two end regions of the racetrack FRC decouple. In contrast, when the elliptic equilibrium is considered (Fig. 1a), the perturbation is concentrated around the magnetic null point, and $n=1$ even mode is stable $^{3}$. 

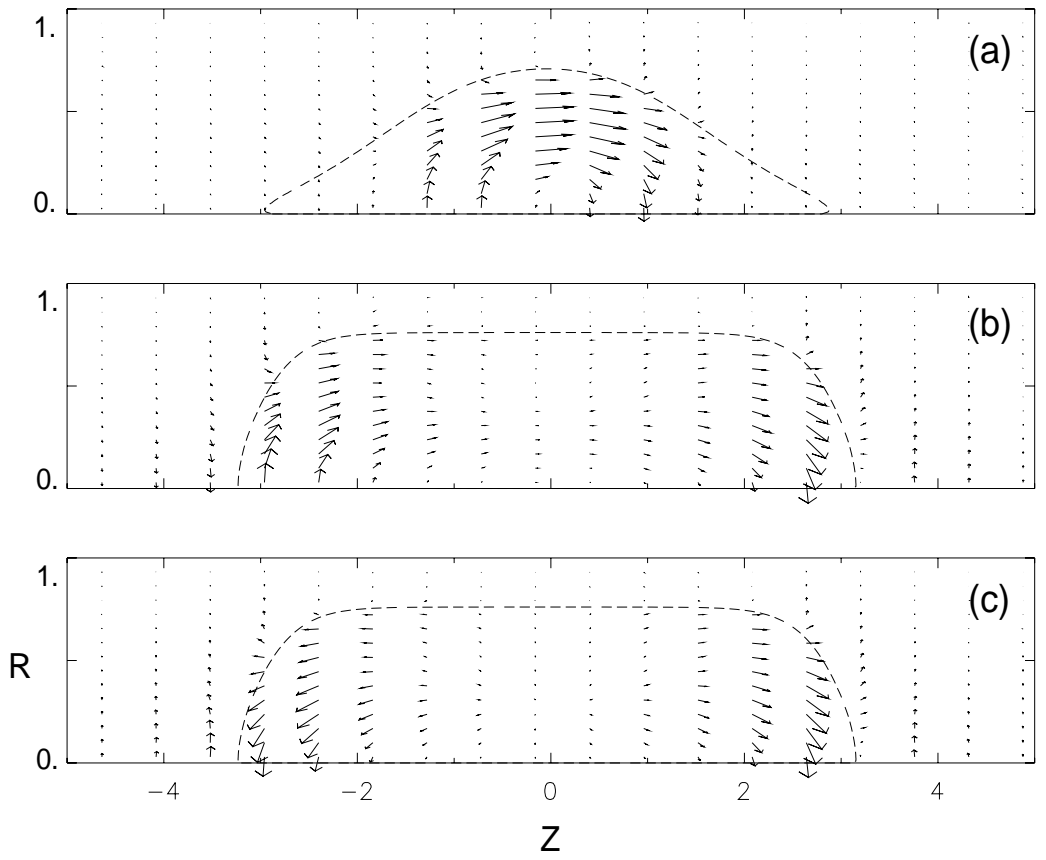

Fig.1: Velocity vectors and the separatrix positions from MHD simulation of the $n=1$ tilt mode $(E=4)$; (a) elliptical separatrix, (b) racetrack separatrix, and (c) $n=1$ even mode with the racetrack separatrix.

Due to the different dependence on the elongation, the relatively long $(E \gtrsim 4)$ configurations with a racetrack separatrix shape generally are more unstable to tilting, than the configurations with an elliptical separatrix. In terms of the growth rate, Eq. (20), the numerical coefficient $C$ is of the order 2.0-3.0 for the racetrack FRCs, and $C \sim 1$ for the elliptical equilibria. The nonlinear evolution of the tilt mode for both elliptical and racetrack equilibria has been investigated in the simulations as well. Fig. 2 illustrates the constant pressure contours on a $2 \mathrm{D}$ plane passing through the symmetry axis at 4 times during the nonlinear growth of the $n=1$ tilt mode in an elliptical equilibrium with $E=4$. It is seen that for most of the growth of the mode, the distortion is mostly internal to the separatrix. 
Only near the end, at $t>10 t_{A}$, does significant distortion to the equilibrium occurs, where $t_{A}=R_{c} / V_{A}$ is the characteristic Alfvén time. At $t>12 t_{A}$, when the tilting motion has "wrapped around" the ends, an axial force imbalance occurs and the two ends repel one another, destroying the configuration.
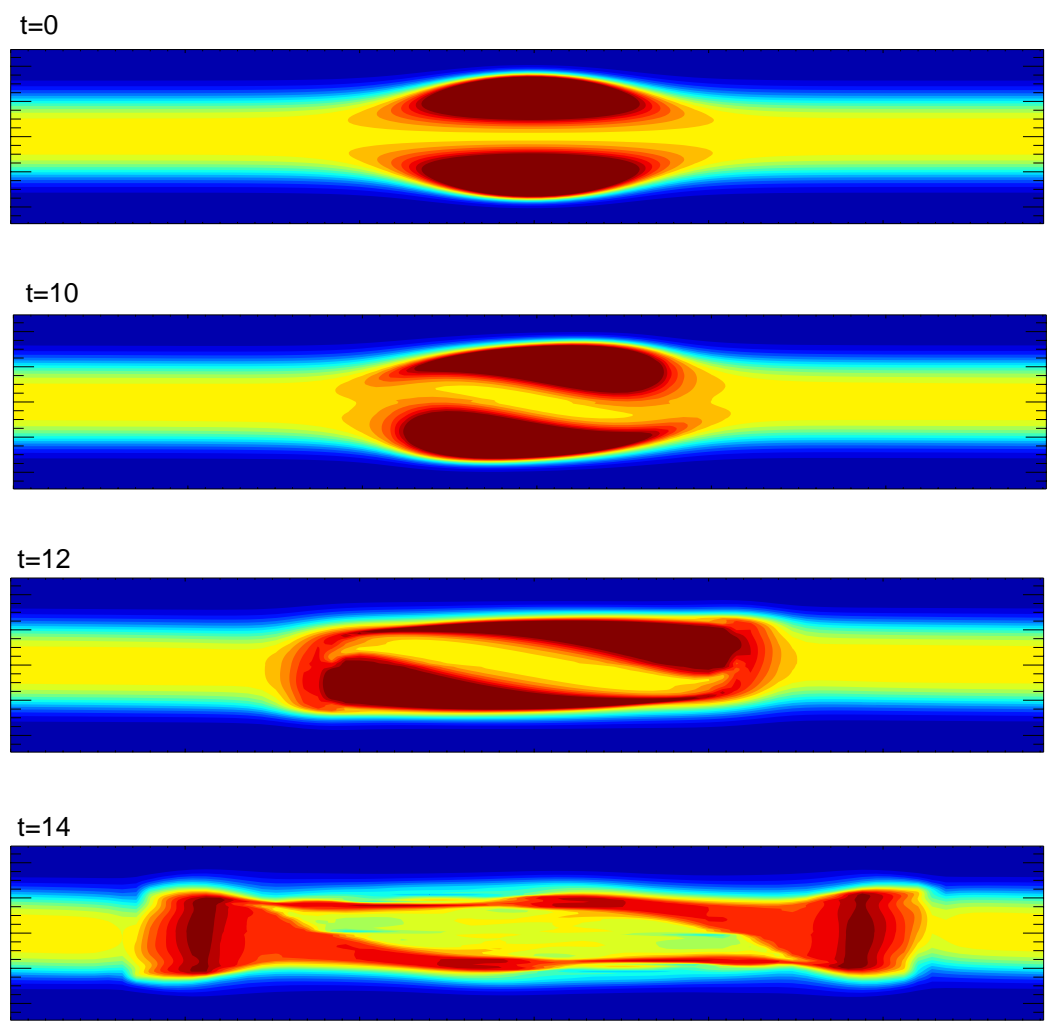

Fig.2: The pressure contours in the poloidal plane at $t=0,10 t_{A}, 12 t_{A}$, and $14 t_{A}$ for the case of $E=4$, elliptic separatrix.

No nonlinear stabilization has been found in either the racetrack or the elliptical configuration. However the nonlinear behavior was somewhat different due to differences in the linear mode structures. For the racetrack equilibrium, the perturbation grows near low-density end regions of the FRC during the linear phase. When the velocity amplitude becomes large enough $\left(\delta V_{z} \sim 0.1-0.3 V_{A}\right)$, the perturbation propagates from the end region to the midplane, pushing along a more dense plasma around the magnetic null. This 
contrasts with the tilt evolution in the elliptical configuration, where most of the plasma inside the separatrix is involved in the tilt motion during the linear growth. As a result, the nonlinear phase of the instability for the racetrack equilibrium is longer than that for the elliptical equilibrium.

\section{B. Sheared rotation effects}

Previous calculations ${ }^{2,7}$ have shown that the tilt instability can be significantly suppressed by plasma toroidal rotation if the rotational Mach number is greater than about 2.0, $M=\omega R_{s} / V_{A} \gtrsim 2$. When a large external mirror field is applied in addition ${ }^{2}$, the growth rate can be strongly reduced by rotation with Mach number $M \gtrsim 1.0$. Although plasma spin up in the toroidal direction has been observed in experiments, such large rotation rates are considered unrealistic. Significant velocity shear, however, is likely to exist in the FRCs, and it has been numerically shown to have a stabilizing effect on the tilt mode ${ }^{2}$. For these reasons, we have investigated the possibility of tilt mode stabilization at smaller rotation rates $(M<1)$ due to velocity shear.

The effect of sheared rotation on the $n=1$ tilt instability was studied by performing MHD simulations with different rotation profiles, separatrix elongations and Mach numbers $M_{s}=V_{s} / V_{A}$, where $V_{s}=\omega R_{s}$ is the rotational velocity at the separatrix at the midplane. Sheared rotation is found to be a more effective stabilizing mechanism for a rotation profile which is peaked near the separatrix and with the angular velocity decreasing to zero at the magnetic null and near the radial wall (Fig. 3). Since the mechanisms leading to FRC rotation are thought to be the loss of particles with a preferential angular momentum or to be end-shorting of the open field lines ${ }^{1}$, it is reasonable to expect a large rotational velocity near the edge region.

The simulations were done for the pressure profile given by $\left(M_{s}=0\right)$ :

$$
p(x)=0.5 p_{0}(\tanh (a x+b)+1), \quad x=\psi / \psi_{0},
$$

where $\psi_{0}$ is the value of the flux at the magnetic null point $\left(\psi_{0}<0\right), a=1.5$ and $b=-1.0$ are the pressure profile parameters, $x>0$ inside the separatrix and $x<0$ outside. The angular velocity profile was taken to be:

$$
\omega(x)=\omega_{0}\left[3(1-x)(1+\tanh (2 x))+0.5\left(x-x_{1}\right)(1-\tanh (2 x))\right]
$$


where $x_{1}=\psi_{w} / \psi_{0}, \psi_{w}$ is the flux at the wall, and $v_{\phi}=r \omega$.
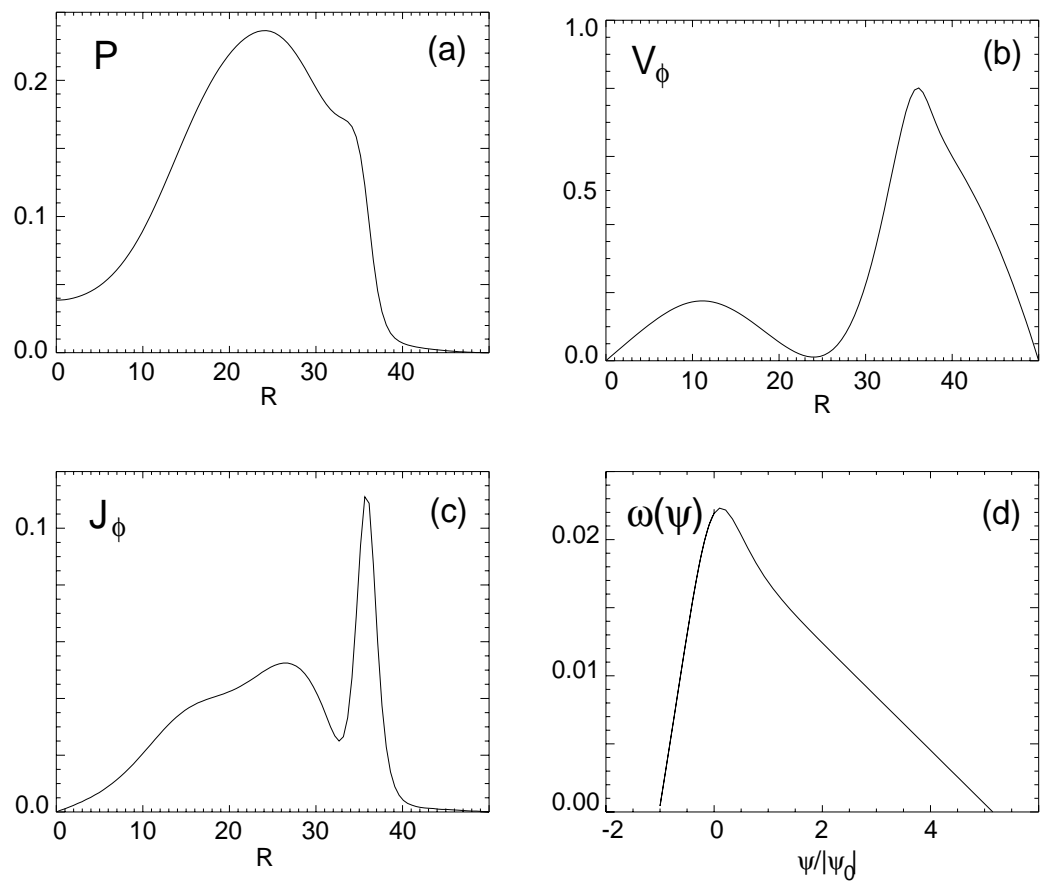

Fig.3: The equilibrium profiles for MHD simulations with sheared rotation: the radial profiles of (a) plasma pressure, (b) toroidal velocity and (c) current at the midplane, and (d) angular rotation velocity versus normalized flux for the equilibrium with $E=2.7$ and $M_{s}=V_{s} / V_{A}=0.8$.

An example of the equilibrium profiles from the MHD simulations with sheared flow is shown in Fig. 3 for a hollow current profile, $E=2.7$ and $M_{s}=0.8$. Shown are the radial profiles of the normalized plasma pressure, current, and toroidal velocity at the midplane $(z=0)$, and angular velocity as a function of flux. Here the pressure is normalized to the external magnetic field pressure, $B_{0}^{2} / 8 \pi$, velocity is normalized to $V_{A}$, and length is normalized to $V_{A} / \omega_{c i}$, where $\omega_{c i}$ is the ion cyclotron frequency in the external field.

A tilt-like initial velocity perturbation of $0.01 V_{A}$ was applied at the beginning of the 
simulation, and the growth rate was calculated from the time evolution of the $n=1$ component of kinetic energy. A grid of $(100 \times 32 \times 150)$ grid points was used in all sheared rotation calculations. The equilibrium parameters and the calculated tilt mode growth rates from the simulations with different elongation are summarized in Table I, where $\gamma_{0}=V_{A} / Z_{s}$.

In Fig. 4, we illustrate the change in the linear mode structure due to shear flow as compared to the non-rotating case. This is shown for both the equilibria with elliptical $(E=2.7)$ and racetrack separatrix shape $(E=6.4)$. The linear mode structure is seen to change in a such way that the perturbation localizes in a region of smaller velocity shear. In the presence of sheared rotation, the tilt mode was observed to rotate with a real frequency comparable to the average rotation frequency. No indication of nonlinear stabilization in the presence of large velocity shear has been found.

In simulations with larger elongation (smaller $\gamma_{0}$ ), the stabilizing effect of the sheared rotation was stronger. In particular, for $E=6.4$ and $M_{s}=0.68$ the growth rate was reduced by a factor of 3 compared to the case without rotation. Our simulations show that, in general, a shearing rate $\Delta \omega=\omega(0)-\omega(1) \gtrsim \gamma_{0}$ is required in order for considerable reduction in the instability growth rate to occur. This means that even in the presence of velocity shear, a large rotation rate, $M_{s} \sim 1$, is still needed to significantly suppress the tilt instability in the MHD regime. It possible, however, that the combination of another effect (FLR, for example) that reduces the growth rate together with velocity shear could lead to complete stabilization of the tilt mode at a more reasonable toroidal velocity.

TABLE I. Parameters for sheared rotation simulations

\begin{tabular}{lccc}
\hline \hline run & $E$ & $M_{s}$ & $\gamma / \gamma_{0}=\gamma Z_{s} / V_{A}$ \\
\hline R1 & 2.4 & 0.0 & 1.32 \\
R2 & 2.7 & 0.63 & 0.89 \\
R3 & 2.7 & 0.8 & 0.54 \\
R4 & 5.6 & 0.0 & 2.7 \\
R5 & 6.4 & 0.68 & 0.9 \\
\hline \hline
\end{tabular}



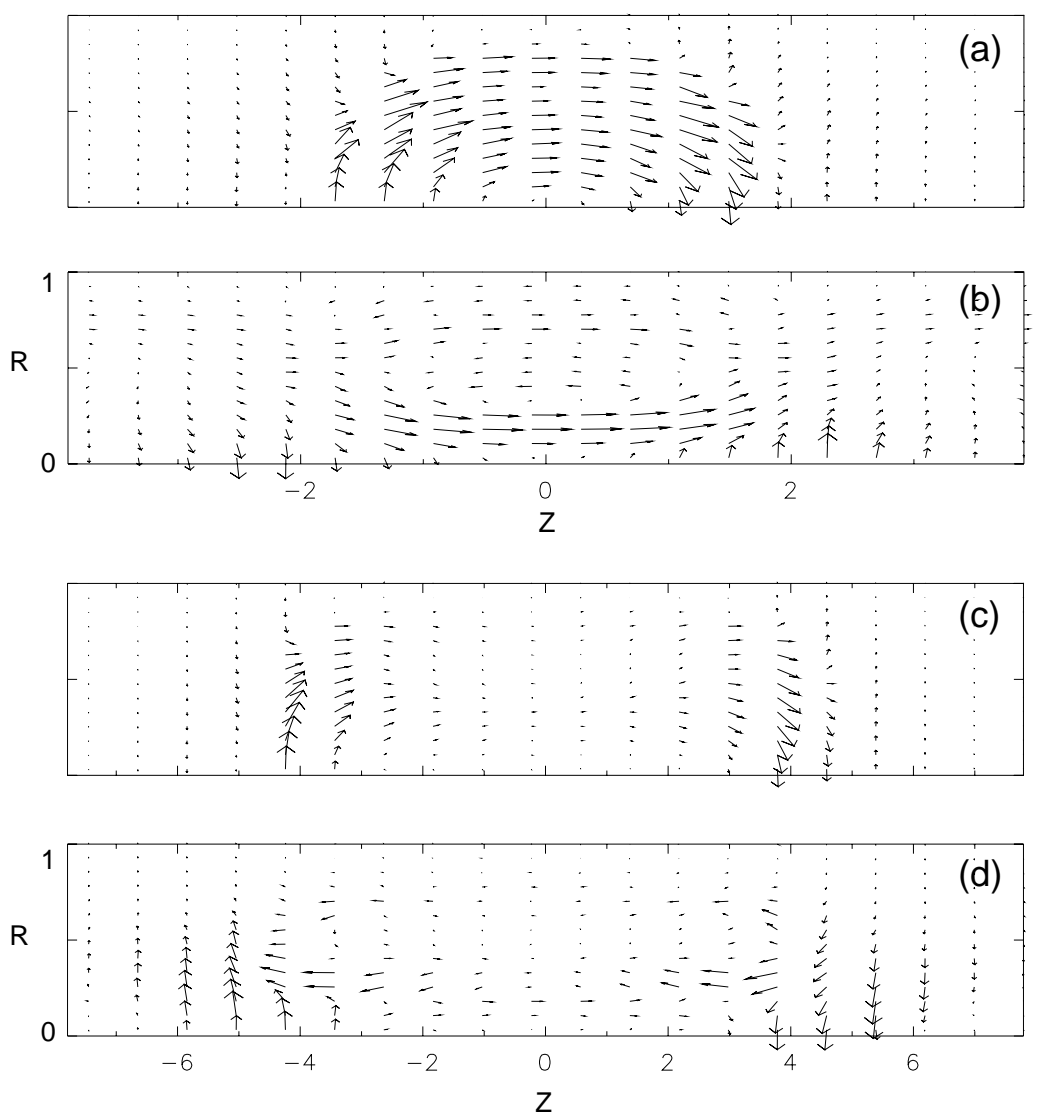

Fig.4: Poloidal mode structure (a) without rotation, $E=2.4$, (b) for $M_{s}=0.8$ and $E=2.7$, (c) without rotation, $E=5.6$, (d) for $M_{s}=0.68$ and $E=6.4$.

\section{HYBRID SIMULATION RESULTS}

We have performed quasi-neutral hybrid simulations of the $n=1$ tilt mode to study how the kinetic effects associated with large thermal ion orbits can modify the MHD predictions. Both linearized and fully nonlinear hybrid models have been implemented in our code. The linearized simulations provide a more accurate result for the instability growth rate and allow simulations of modes other than the most unstable one (with different $n$ ). In addition, a smaller number of simulation particles and field substeps are required for numerical convergence and stability. The nonlinear version of the hybrid scheme is described in Section 
II. In the linearized version, the particle trajectories are calculated in the equilibrium field, the nonlinear term proportional to $w$ is dropped on the right hand side of the equation for particle weight, Eq. (11), and a linearized form of the Ohm's law, Eq. (10), is used.

The vacuum region is treated in a way similar to that for the MHD simulations. The simulation particles are initially loaded by inverting the $2 \mathrm{D}$ ion density profile given by Eq. (19) (for the exponential rigid rotor distribution) or by $n_{i}=p_{i} / T_{i}$ (for a Maxwellian distribution), with a negligible density in the vacuum region and near the radial wall. The value of the ion density actually used in the $3 \mathrm{D}$ stability calculations is taken to be the maximum of that calculated and a cutoff density, normally chosen to be $n_{c}=10 \%$ of the peak density. In some cases this method turned out to be numerically unstable. In these cases the simulation particles were loaded with a density value that corresponds to adding an offset value of $n_{c}$ to the equilibrium density. At constant temperature, this corresponds to adding a small constant pressure to the equilibrium pressure profile and does not change the equilibrium equation. In both cases the convergence of the results with respect to the value of the cutoff density has been checked. The conservation of the total energy and the number of particles ${ }^{27} \delta N / N=\left(\sum_{m} w_{m}\right) / N$ has been monitored in the nonlinear $\delta f$ runs as an accuracy check and for debugging purposes.

\section{A. Test cases}

Several tests have been done to benchmark our hybrid version of the code. First, the simulations of the tilt mode in FRC with elongation $E=3.9$ have been performed using both the nonlinear $\delta f$ scheme and the full $f$ particle simulations in order to verify the $\delta f$ scheme. The Maxwellian distribution function was used for ions $\left(F_{0}=F_{0}(\varepsilon)\right)$, and a relatively large $\bar{s}(\bar{s}=7.4)$, MHD-like regime was considered, so that comparison could be made with MHD results as well. Fig. 5 shows the energy plots from these three different simulation runs: MHD, hybrid $\delta f$ and hybrid full $f$ simulations. The same equilibrium profiles were used in all three cases. A $(100 \times 32 \times 150)$ cylindrical grid was used in the MHD simulations, $(50 \times 32 \times 60)$ cylindrical grid for fields and $(51 \times 51 \times 60)$ Cartesian grid for particles were used in the hybrid simulations. The number of the simulation particles was 1,000,000 and $2,000,000$ in the $\delta f$ and full $f$ runs respectively. 


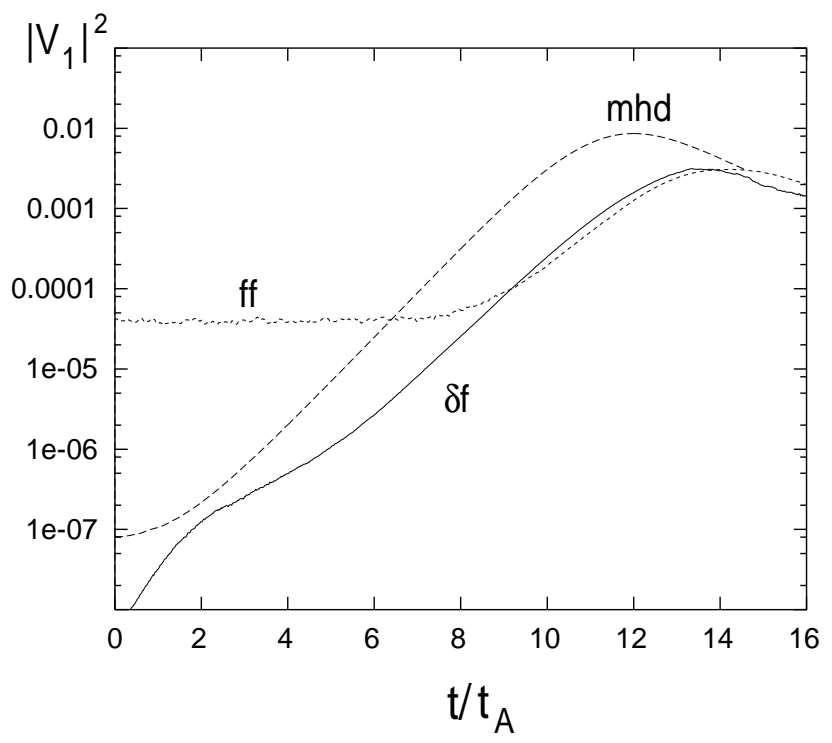

Fig.5: The temporal evolution in the $n=1$ component of kinetic energy from $M H D, \delta f$ hybrid and full $f$ hybrid simulations all done for the same equilibrium profiles with $E=3.9$ and $\bar{s}=7.4$.

Note that in Fig. 5, the squares of the amplitude of the $n=1$ Fourier component of $\mathbf{V}$ integrated over the $r-z$ volume and normalized to the equilibrium magnetic field energy are plotted, where $\mathbf{V}$ is the fluid velocity for MHD simulation, $\mathbf{V}=\mathbf{V}_{\mathbf{i}}$ is the ion fluid velocity for hybrid runs. The time is normalized to the Alfvén time, which is defined as the ratio of the cylinder radius to the characteristic Alfvén speed, $t_{A}=R_{c} / V_{A}$.

There is very good agreement in the linear growth rate and the mode structure in the $\delta f$ and full $f$ simulation runs. The growth rate from hybrid simulations is slightly smaller than that from MHD run due to kinetic effects. Similar nonlinear evolution was observed in the both hybrid runs as well. However, the numerical noise level in the full $f$ simulation was rather high, and no growth of the tilt mode could be seen in the energy plot until $t>8 t_{A}$ because of the numerical noise.

As another test, a $\delta f 2 \mathrm{D}$ hybrid simulation of the $n=2$ rotational mode was done 
for equilibrium profiles used in a previous work ${ }^{28}$. In agreement with the previous results, the growth of the dominant $n=2$ mode was observed for the equilibrium with $60 \%$ of field reversal, separatrix beta $\beta_{s}=0.5$ and the rotational parameter ${ }^{28} \alpha=1$ (no electron rotation).

The accuracy of the particle pushing and the interpolation schemes was checked by following the unperturbed particle orbits in the equilibrium field for a representative FRC equilibrium with $E=3.9$ and $\bar{s}=1.9$. The conservation of the particle energy, $\varepsilon=m v^{2} / 2+$ $e \phi$, and the toroidal angular momentum, $p_{\phi}=m r v_{\phi}-e \psi / c$, was monitored for several particle trajectories for long times. Fig. 6a shows a typical particle orbit projected on the poloidal plane, and Figs. 6b,c show the variation of the particle energy and the angular momentum along this orbit.
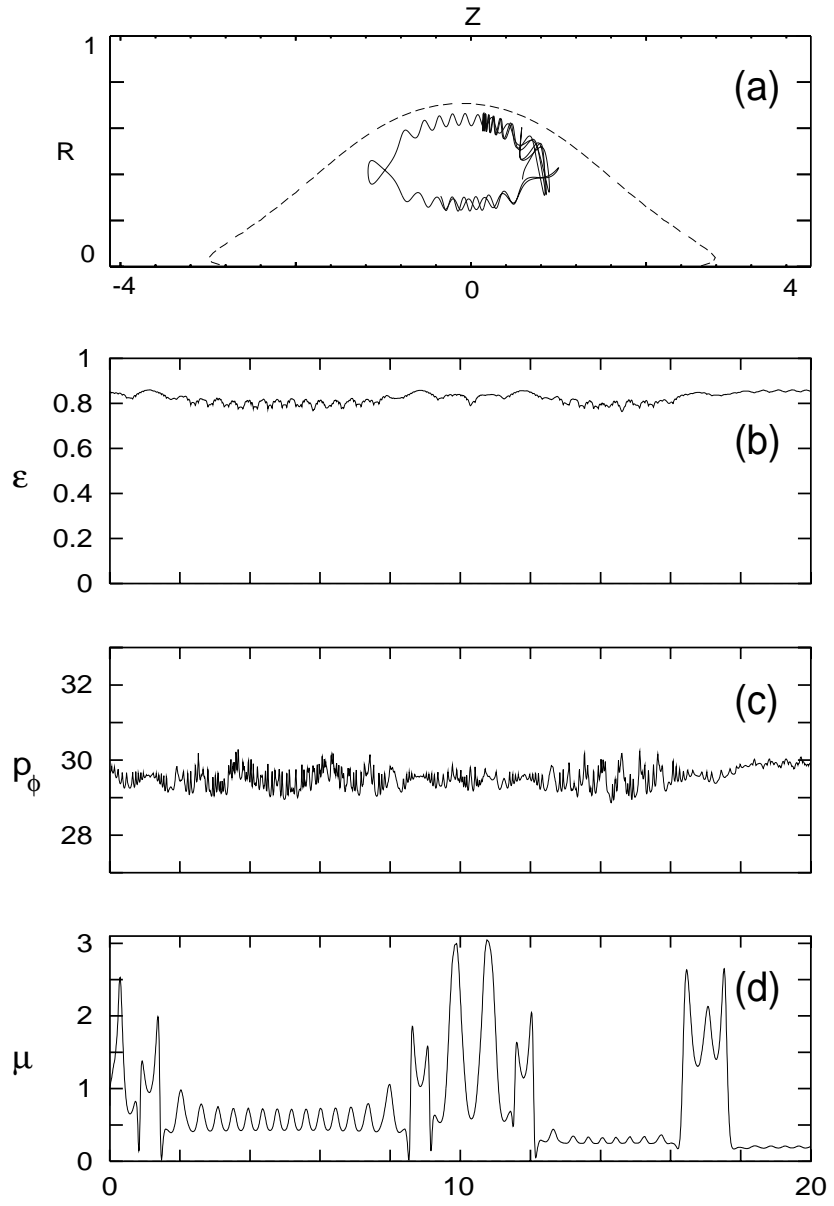

Fig.6: Poloidal projection of the typtcal equilibrium orbit (a); time variation of (b) particle total energy; (c) toroidal angular momentum; (d) magnetic moment along this orbit. 
The relative amplitudes of the fluctuation in $\varepsilon$ and $p_{\phi}$ are $\sim 10 \%$ and $\sim 4 \%$ respectively without any secular changes. Also shown on Fig. 6d is a variation of the magnetic moment, $\mu$, calculated in the rotating frame with zero equilibrium electric field. It is seen that the magnetic moment is not conserved along this trajectory, and it undergoes large changes when the particle passes through the large curvature regions.

\section{B. Linear stability of the $n=1$ tilt mode}

A number of hybrid simulation runs have been done starting from different kinetic equilibria in order to investigate the dependence of the growth rate of the tilt instability on the kinetic parameter $\bar{s}$, the elongation and the shape of the separatrix. The equilibrium parameters and the linear growth rates from some of these simulations are listed in Table II, where the notation is as follows: $x_{s}$ is the ratio of the separatrix radius to the cylinder radius, $\beta_{s}$ and $M_{s}$ are the plasma beta and rotational Mach number respectively calculated at the separatrix at the midplane, $\gamma_{m h d}$ is the growth rate calculated in the MHD simulations for the same equilibria, and $\gamma_{0}=V_{A} / Z_{s}$. The Mach number is calculated as $M_{s}=V_{i} / V_{A}$, where $V_{i}$ is the ion fluid velocity.

TABLE II. Parameters for hybrid simulations

\begin{tabular}{lcccccccc}
\hline \hline run & $E$ & $x_{s}$ & $\beta_{s}$ & $M_{s}$ & shape & $\bar{s}$ & $\gamma_{m h d} / \gamma_{0}$ & $\gamma / \gamma_{0}$ \\
\hline RH1 & 2.0 & 0.76 & 0.19 & 0.09 & elliptic & 9.2 & 1.5 & 1.4 \\
RH2 & 1.7 & 0.78 & 0.17 & 0.55 & elliptic & 1.5 & 1.5 & 0.98 \\
RH3 & 3.9 & 0.71 & 0.30 & 0.0 & elliptic & 7.4 & 1.8 & 1.6 \\
RH4 & 3.9 & 0.71 & 0.30 & 0.0 & elliptic & 1.9 & 1.8 & 0.73 \\
RH5 & 3.9 & 0.71 & 0.30 & 0.0 & elliptic & 0.9 & 1.8 & 0.34 \\
RH6 & 4.1 & 0.76 & 0.15 & 0.0 & racetrack & 1.4 & 2.2 & 0.97 \\
RH7 & 7.2 & 0.64 & 0.33 & 0.0 & racetrack & 6.2 & 2.54 & 2.01 \\
RH8 & 7.2 & 0.64 & 0.33 & 0.0 & racetrack & 0.8 & 2.54 & 0.34 \\
\hline \hline
\end{tabular}


The first two runs in the Table II, RH1 and RH2, were done for equilibria with no electric field and the exponential rigid-rotor distribution function, Eq. (18), was used for the ions. These equilibria have a relatively small elongation, $E \sim 2$, peaked current profile and an elliptic separatrix shape. Note that for the exponential rigid-rotor equilibria, a small value of $\bar{s}$ corresponds to a large rotation Mach number, which can have an additional stabilizing effect.

In the rest of the runs, the Maxwellian distribution function $F_{0}=F_{0}(\varepsilon)$ was used for the ions with $\mathbf{J}_{\mathbf{i}}=0$ and all the current was carried by the electrons. The simulations with elongation $E \sim 4$, were done for equilibria with both elliptic and racetrack separatrix shapes in order to investigate the effectiveness of the FLR stabilization for different configuration shapes and eigenmode structures. In the simulations RH3-RH5 the pressure profile was taken to be $\mathrm{be}^{24}$

$$
p(\psi)=p_{0} \begin{cases}K_{0}+x-d / 2 x^{2}, & x>0 \\ K_{0} \exp \left(x / K_{0}\right), & x \leq 0\end{cases}
$$

where $x=\psi / \psi_{0}(x>0$ inside the separatrix $), \psi_{0}$ is the value of the flux at the magnetic null point, $K_{0}$ is a numerical parameter proportional to the value of the plasma beta at separatrix, and $d$ is the profile hollowness parameter. Simulations were carried out for $\beta_{s}=0.3$, and $d=-0.5$. In the simulations with the racetrack separatrix, RH6-RH8, the ion pressure profile was as in Eq. (21) with the numerical parameters equal to $a=1.7$ and $b=-1.0$ for the RH6 equilibrium, and $a=1.2$ and $b=-0.5$ in the runs with $E=7.2$.

The initial $n=1$ perturbation in the axial component of the ion fluid velocity $\left(\sim 0.01 V_{A}\right)$ was applied at $t=0$ by assigning a finite weight $w \sim v_{z}$ to the particles inside the separatrix. The linear growth rate was calculated from the time evolution of the amplitude of the $n=1$ component of electron velocity, $\left|V_{e 1}\right|^{2}$, integrated over $r-z$ volume. The linear stability results for two family of equilibria with $E \approx 2$ and $E=7.2$ are summarized in Fig. 7 , where the growth rate is shown for different values of $1 / \bar{s}$. The growth rates are normalized to the corresponding MHD growth rates. Notice that $\gamma$ is only slightly reduced for $\bar{s} \geq 1.5(1 / \bar{s} \leq 0.66)$ in the simulations with small elongation $E \sim 2$, in agreement with previous results $^{11,12}$. These simulations $(E \approx 2)$ were performed for the exponential rigidrotor ion distribution function, as RH1 and RH2 runs, and the ion rotation Mach number 
was $M \leq 0.55$ for $\bar{s} \geq 1.5$. Since previous MHD simulations have shown that a stabilizing influence of rigid rotation should be felt at a much larger rotation rate $^{2}$, the reduction in the growth rate seen in Fig. 7 is mostly due to FLR effects. The simulations with larger elongations were performed for Maxwellian ion distribution function with zero ion rotation. For the configurations with $E=7.2$, there is a significant reduction in the tilt instability growth rate at small values of $\bar{s}(\bar{s} \sim 1)$, however, no absolute stabilization has been found for $\bar{s} / E$ values as small as 0.1 .

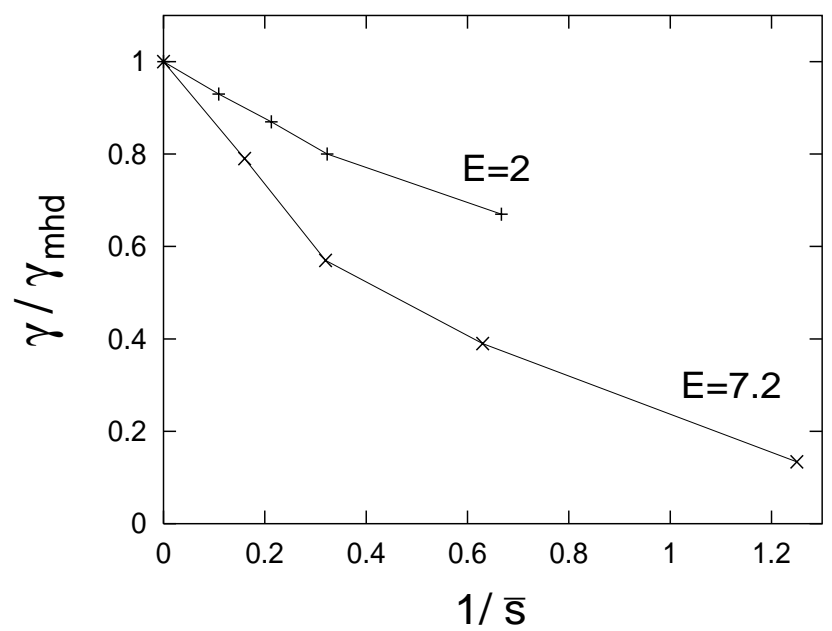

Fig.7: Variation of the normalized growth rate of the $n=1$ tilt instability with $1 / \bar{s}$ parameter. The points obtained in the linearized hybrid simulations for two different elongations, $E \approx 2$ and $E=7.2$, are indicated.

The previous kinetic calculations by Barnes et al. ${ }^{6}$ based on a Vlasov-fluid dispersion functional approach and trial functions found a strong reduction in the tilt mode growth rate for $\bar{s} \sim 2$ and complete stabilization at $\bar{s} \lesssim 1.5$ for an equilibrium with elliptical separatrix and $E=7.7$. Our simulations with different separatrix shapes (see Table II) show that there is no dramatic difference in the FLR stabilization between the equilibria with different 
separatrix shapes. In particular, at $\bar{s}=1.4$ and $E \approx 4$ the growth rate was reduced by a factor of 2.3 in the simulations with the racetrack separatrix, and a factor of 3 reduction was obtained for the elliptic equilibrium. The difference between our results and the dispersion analysis results ${ }^{6}$, therefore, must be due to the deviation of the true kinetic eigenfunction from the assumed MHD-like trial function ${ }^{6}$ in the strongly kinetic regime (small $\bar{s}$ ).

Our simulations demonstrate that the linear structure of the tilt mode changes as the value of $\bar{s}$ is reduced. Figs. 8 and 9 show poloidal and toroidal mode structures from the hybrid simulations with different values of $\bar{s}$, along with the corresponding MHD eigenmode. These simulations were performed for equilibria with elongation $E=3.9$ and with elliptical separatrix shape. Shown are vector plots of the poloidal component (Fig. 8) and contour plots at the midplane (Fig. 9) of the axial component of the ion fluid velocity (for the hybrid runs) and fluid velocity (for the MHD run).

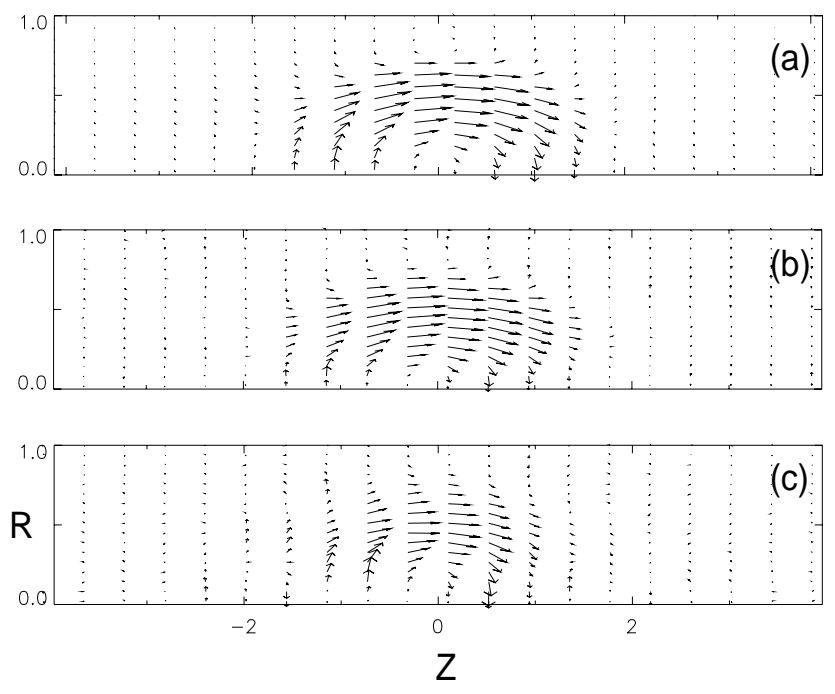

Fig.8: Vector plots of poloidal component ( $r-z$ plane) of the ion fluid velocity: (a) from the MHD simulation, (b) and (c) from the hybrid simulations with $\bar{s}=7.4$ and $\bar{s}=0.9, E=3.9$. 
For the larger value of $\bar{s}(\bar{s}=7.4$, Fig. $8 \mathrm{~b})$, both the growth rate and the eigenfunction are close to that of the MHD result (Fig. 8a). The mode structure is seen to change in the kinetic regime with $\bar{s}=0.9$. In particular, the perturbation becomes more localized near the magnetic null point (Fig. 8c), the mode rotates $\left(\omega_{r} \neq 0\right)$, and it has a more complicated toroidal structure (Fig. 9c).
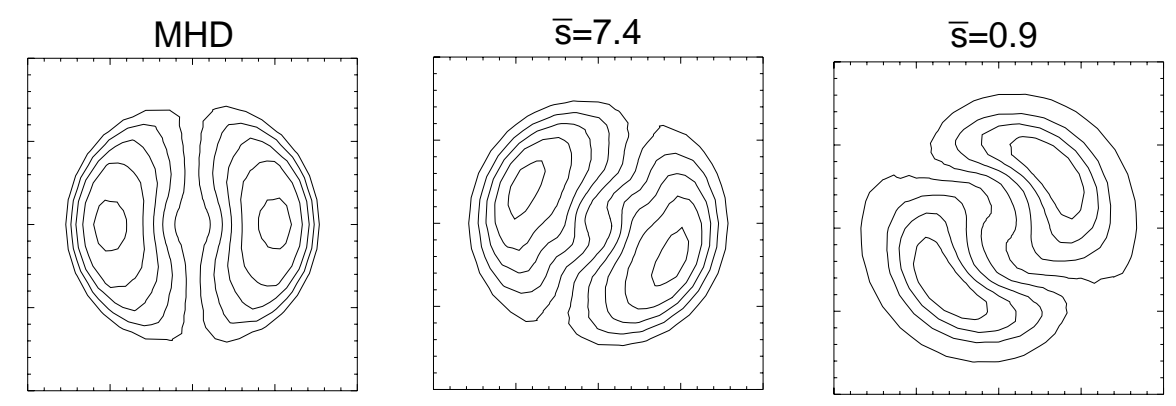

Fig.9: Contour plots of the axial component (at the midplane) of the ion fluid velocity: (a) from the MHD simulation, (b) and (c) from the hybrid simulations with $\bar{s}=7.4$ and $\bar{s}=0.9$, same simulations as on Fig.8.

The kinetic results presented in Table II and in Fig. 7 have been obtained with the linearized hybrid simulations. In these runs, a $(60 \times 32 \times 80)$ cylindrical grid for the fields and a $(51 \times 51 \times 80)$ Cartesian grid for the particles were used, and the number of simulation particles was between $0.25 \cdot 10^{6}$ and $0.5 \cdot 10^{6}$. The particle time step was set by $d t=0.1 / \omega_{c i}$, and 1 to 16 field substeps per particle step were done, depending on the value of $\bar{s}$. The convergence of the results with respect to the time step, grid size, the number of the simulation particles and the value of the cutoff density, $n_{c}$, has been verified in the simulations. For the set of parameters used in the simulations, the tilting instability growth rate was affected mostly by the value of $n_{c}$. Namely, the value of $\gamma$ was increased by several percent as $n_{c} / n_{0}$ was reduced from 0.1 to 0.05 . No significant difference in the growth rate value or the linear mode structure has been found in the simulation runs performed with better resolution or smaller time step. The results obtained for the tilt mode from the linearized simulations in 
this study (with $E>1$ ) were also not sensitive to the value or the profile of the resistivity.

\section{DISCUSSIONS AND CONCLUSIONS}

Both MHD and hybrid simulations were performed in order to study the $n=1$ tilting instability in prolate FRCs. Profile effects, sheared rotation, and kinetic effects on the tilt mode stability were considered.

Our MHD simulations show that the dependence of the tilt mode growth rate on elongation for a racetrack equilibrium is different from the previously believed inverse scaling. This result is in agreement with a recent spectral stability study ${ }^{3}$. It has also been shown that for a racetrack separatrix shape and large enough elongation $(E \gtrsim 3)$, the tilt mode is localized near the FRC end regions, where the curvature is large, and the perturbation growths in the two end regions decouple from one another. As a result, the growth rate is nearly independent of $E$, and another mode with even symmetry relative to the midplane, appears and is unstable with its growth rate close to that of the tilt mode. This contrasts sharply with the usually assumed "rigid tilt" model, in which the (stabilizing) inertial term increases with the elongation, and reduces the growth rate.

This result is important both when comparing theoretical and experimental stability, and also when assessing the effect of different stabilizing factors. The weaker dependence of the MHD growth rate on elongation implies a change in the previously assumed stable parameter regimes ${ }^{4,8}$. In particular, we agree with other recent work ${ }^{10}$ that claims that the previously proposed stability scaling with $\bar{s} / E$ is not valid. The breakdown of this scaling has important implications for estimating when FLR effects might lead to tilt stabilization.

The possibility of tilt mode stabilization by sheared flows has been investigated in the MHD simulations as well. The sheared toroidal rotation is found to reduce the growth rate of the tilt mode, however a large rotation rate, $\Delta V \sim V_{A}$, is still required to significantly affect the mode growth, and complete stabilization has not been found. It is possible that a combination of some other stabilizing effect (FLR, for example) and velocity shear will lead to tilt mode stability at realistic rotation rates, but this has not been demonstrated.

Our MHD simulations have shown no indication of nonlinear saturation for all separatrix shapes and pressure profiles considered. This is consistent with previous calculations ${ }^{2}$. The 
mode structure is calculated to be primarily internal far into the nonlinear regime, making it difficult to detect experimentally. The mode finally destroys the configuration by wrapping around at the ends to create an axial force imbalance, causing the FRC to break apart. We note here that in the MHD regime the $n>1$ modes have growth rates larger than that of the $n=1$ mode, and may destroy the configuration before the $n=1$ tilt could grow to a noticeable amplitude. This would further hamper experimental detection of an unstable tilt mode.

Since most of the FRC experiments to date have been in the kinetic regime, with the ion Larmor radius comparable to that of the separatrix radius (small to moderate value of the kinetic parameter $\bar{s}$ ), kinetic effects are considered to be a major factor in the observed FRC stability. We have performed 3D hybrid simulations in order to study the thermal ion kinetic effects on the tilt mode in the prolate FRC. We have included ion kinetic effects self-consistently, and studied the stability of several experimentally realistic equilibria (with large $E$ ). This paper concentrates on the linear stability of the $n=1$ tilt mode in the kinetic regime with zero velocity shear, and the results of the nonlinear hybrid simulations will be presented elsewhere. The $\delta f$ method $^{21}$ has been used to greatly reduce simulation noise and computational requirements, and has been found particularly useful in the linear stability study.

Our hybrid simulations show that for large $\bar{s}(\bar{s} \sim 5-10)$ the growth rate and the linear mode structure of the kinetic tilt mode are very close to that predicted by MHD. However, as the value of $\bar{s}$ is decreased to $\bar{s} \sim 1-2$ the growth rate is reduced, and this reduction is stronger for configurations with larger elongation $(E \gtrsim 4)$. In particular, an order of magnitude reduction in $\gamma$ has been obtained in the simulations with $E=7.2$ and $\bar{s}=0.8$. It also has been shown that the linear mode structure of the tilt mode changes from that predicted by MHD in the small $\bar{s}$, kinetic regime. No strong dependence of the kinetic stabilization on the separatrix shape has been found, and similar results have been obtained in the simulations with elliptical and racetrack equilibria for $E \sim 4$. Several simulation runs have been performed with different kinetic equilibrium profiles. In all cases with $E \gtrsim 4$, considerable reduction in the tilt growth rate was obtained for $\bar{s} \lesssim 2$. However, no absolute stabilization of the tilt instability has been found even for very small values of $\bar{s}$. 
Our results are different from those of previous kinetic calculations based on a Vlasovfluid dispersion functional and trial function approach ${ }^{6}$. In these studies, a much stronger reduction in the growth rate (factor of 10-15) was obtained for $\bar{s} \lesssim 3$ and complete tilt mode stabilization was found for $\bar{s} \lesssim 1.5(E=7.7)$. The difference between our results and the dispersion analysis results can be explained by the deviation of the true self-consistent kinetic eigenfunction from the assumed MHD-like trial function used by Barnes et al. ${ }^{6}$, in the strongly kinetic regime.

The non-ideal MHD and kinetic effects included in our hybrid model are: two-fluid effects (Hall term in the Ohm's law), finite ion Larmor radius effects, and ion resonant effects. The inclusion of the Hall term results in the magnetic field being frozen into the electron fluid, and causes the ion perpendicular motion to be different from that of the electrons. Due to the finite size of the Larmor orbits, the ions drift in the gyro-averaged field, and therefore FLR also produces ion motion relative to the electrons. This causes the tilt mode to rotate and have a non-zero real frequency, comparable to the ion diamagnetic frequency.

Several previous studies have considered the Hall term and FLR effects in the two-fluid and in the small ion Larmor radius (gyroviscous effect) approximations ${ }^{2,4,5,8}$. The physical mechanisms for the FLR and the Hall term stabilization are similar and have been identified as the phase shift between the electron and ion displacements, which results in the magnetic and fluid perturbation being out of phase, and leads to mode suppression ${ }^{4}$. Although the earlier semi-analytic estimates ${ }^{4}$ gave optimistic results indicating stabilization of the tilt mode at small $\bar{s}$, the later more self-consistent calculations found that the stabilizing effect was not as strong as expected ${ }^{2,5}$, mostly due to the change in the linear mode structure at small $\bar{s}$. Our results agree with these calculations in that, although we have found that the $n=1$ tilt instability linear growth rate is reduced by kinetic effects, this reduction (by a factor of 3-5) is not strong enough to explain the stability observed in the FRC experiments for over 100 Alfvén times.

Note that the fluid models are valid only in the moderate to large $\bar{s}$ limit. The important effect missing from these models is the wave-particle resonant interaction, which can be important in the strongly kinetic FRCs. In addition, at small values of $\bar{s}$, most of the ions 
execute very complex motion with a large fraction of their orbits being stochastic, and their magnetic moment not conserved. This makes the perturbative treatment of FLR effects difficult and inaccurate. An advantage of the initial value particle simulations is that all the relevant kinetic effects are included in a self-consistent and non-perturbative way. In particular, the resonant effects and the stochastic ion orbits can be an important factor in maintaining instability in the large Larmor radius cases ${ }^{29,30}$. It has been known for some time that the Vlasov-fluid plasma has the same marginal stability condition as the MHD model, and therefore, that FLR effects cannot totally stabilize, but can only reduce growth rates of unstable modes ${ }^{30,31}$. The residual instability is due to the resonant-particle destabilization of FLR-stabilized MHD modes. In the case of the energetic ion rings, for example, it was shown that a low-frequency instability can exist as a hydromagnetic non-resonant mode in the MHD unstable limit. Two waves with opposite signs for their real frequency are present in MHD stable configurations. The wave propagating in the direction of the toroidal current (the negative energy wave) is destabilized via the inverse Landau damping mechanism. It is possible that a similar resonant instability, driven by the interaction of the wave with the ions for which Doppler shifted frequency matches the betatron frequency $\omega-n \Omega= \pm \omega_{\beta}$, is present in the more general case in the other field-reversed configurations, and is responsible for the finite growth rate obtained in the very small $\bar{s}$ cases in our study.

Finally, our simulations indicate that the apparent FRC stability observed in the experiments cannot be explained within linear theory in the hybrid model. This model assumes cold fluid electrons and fully kinetic ions. Missing from this model are effects such as finite electron pressure, collisions, ion sheared rotation, and non-fluid behavior of the electrons trapped near the magnetic x-points and executing fast curvature drift motion. It is possible that these effects can have an additional stabilizing influence. Another possibility is that the nonlinear behavior of the tilt mode in the kinetic regime is different from that of the MHD prediction. Preliminary results of our nonlinear hybrid simulations at low values of $\bar{s}$ indicate that the instabilities in the prolate configurations saturate through a combination of a lengthening of the initial equilibrium and a modification of the ion distribution function. These results will be reported in a future publication. Note that nonlinear stabilization of a linearly unstable tilt mode may explain the observation in the low $\bar{s}$ experiments of initial 
$n=1$ tilt motion that does not result in total loss of the confinement ${ }^{15}$.

In summary, we have investigated the effects of the separatrix shape, sheared rotation (in MHD regime), and ion FLR on the $n=1$ tilt mode instability. Substantial reduction of the growth rate due to ion sheared rotation and kinetic effects has been found. However, our simulations have shown that for realistic experimental parameters, none of these effects by themselves results in a linearly stable FRC configuration.

\section{ACKNOWLEDGEMENTS}

The authors acknowledge many useful discussions with J. Finn, Y. Omelchenko,

W. Park, L. Steinhauer, and M. Tuszewski. This work was supported by DOE contract \#DE-AC02-76-CHO-3073. 


\section{REFERENCES}

${ }^{1}$ M. Tuszewski, Nucl. Fusion 28, 2033 (1988).

${ }^{2}$ R. D. Milroy, D. C. Barnes, R. C. Bishop, R. B. Webster, Phys. Fluids B 1, 1225 (1989).

${ }^{3}$ N. Iwasawa, A. Ishida and L. C. Steinhauer, J. Phys. Soc. Jpn., 69, 451 (2000).

${ }^{4}$ A. Ishida, H. Momota and L. C. Steinhauer, Phys. Fluids, 31, 3024 (1988).

${ }^{5}$ N. Iwasawa, A. Ishida and L. C. Steinhauer, in Proceedings of The US-Japan Workshop on Physics of High Beta Plasma Confinement in Innovative Fusion Systems (Dec. 14-15 1998, NIFS, Toki, Japan), p. 1.

${ }^{6}$ D. C. Barnes, J. L. Schwarzmeier, H. R. Lewis, C. E. Seyler, Phys. Fluids 29, 2616 (1986).

${ }^{7}$ R. Horiuchi and T. Sato, Phys. Fluids B 1, 581 (1989).

${ }^{8}$ H. Ji, M. Yamada, R. Kulsrud, N. Pomphrey, and H. Himura, Phys. Plasmas 5, 3685 (1998).

${ }^{9}$ J. L. Staudenmeier, Ph.D. thesis, Pennsylvania State University 1991.

${ }^{10}$ N. Iwasawa, A. Ishida and L. C. Steinhauer, Phys. Plasmas, 7, 1 (2000).

${ }^{11}$ K. Nishimura, R. Horiuchi and T. Sato, Phys. Plasmas, 4, 4035 (1997)

${ }^{12} \mathrm{Yu}$. A. Omelchenko, Kinetic simulations of the formation and stability of the Field-Reversed Configuration, to appear in Phys. Plasmas (2000).

${ }^{13}$ E. J. Horowitz, D.E. Shumaker, and D.V. Anderson, J. Comput Phys., 84, 279 (1989).

${ }^{14}$ D. C. Barnes and R. D. Milroy, Phys. Fluids B 3, 2609 (1991).

${ }^{15}$ M. Tuszewski, D. P. Taggart, R. E. Chrien, D. J. Rej, R. E. Siemon, B. L. Wright, Phys. Fluids B 3, 2856 (1991).

${ }^{16}$ A. L. Hoffman, L. N. Carey, E. A. Crawford et al., Fusion Technology 23, 185 (1993).

${ }^{17}$ J. T. Slough and A. L. Hoffman, Phys. Fluids B 5, 4366 (1993). 
${ }^{18}$ T. R. Jarboe, I. Henins, H. W. Hoida, R. K Linford, J. Marshall, D. A. Platts, and A. R. Sherwood, Phys. Rev. Lett., 45, 1264 (1980).

19 G. C. Goldenbaum, Physica Scripta, T2, 359 (1982).

${ }^{20}$ M. Yamada, R. Ellis, Jr., H. P. Furth et al., in Plasma Physics and Controlled Nuclear Fusion Research, (International Atomic Energy Agency, Vienna, 1983), Vol. II, p. 265.

${ }^{21}$ S. E. Parker and W. W. Lee, Phys. Fluids 5, 77 (1993).

${ }^{22}$ E. V. Belova, S. C. Jardin, H. Ji, M. Yamada, R. M. Kulsrud, Bull. Am. Phys. Soc. 44, 85 (1999).

${ }^{23}$ P. N. Guzdar, J. F. Drake, D. McCarthy, A. B. Hassam, C. S. Liu, Phys. Fluids, 5, 3716 (1993).

24 J. W. Cobb, T. Tajima, D. C. Barnes, Phys. Fluids B 5, 3227 (1993).

${ }^{25}$ B. Marder and H. Weitzner, Plasma Physics 12, 435 (1970).

${ }^{26}$ D. W. Hewett and R. L. Spencer, Phys. Fluids 26, 1299 (1983).

${ }^{27}$ E. V. Belova, R. E. Denton, A. A. Chan, J. Comput. Phys., 136, 324 (1997).

${ }^{28}$ D. S. Harned, Phys. Fluids 26, 1320 (1983).

${ }^{29}$ J.M. Finn and R. N. Sudan, Phys. Fluids 22, 1148 (1979).

${ }^{30}$ C. E. Seyler and J. P. Freidberg, Phys. Fluids 23, 331 (1980).

${ }^{31}$ H. Tasso, in Plasma Physics and Controlled Nuclear Fusion Research, (International Atomic Energy Agency, Vienna, 1977), Vol. III, p. 371. 
The Princeton Plasma Physics Laboratory is operated by Princeton University under contract with the U.S. Department of Energy.

\author{
Information Services \\ Princeton Plasma Physics Laboratory \\ P.O. Box 451 \\ Princeton, NJ 08543
}

Phone: 609-243-2750

Fax: 609-243-2751

e-mail: pppl_info@pppl.gov

Internet Address: http://www.pppl.gov 